\title{
Occupational Mobility Patterns: A Case Study of Leadership and Access in the National Football League
}

\author{
Carlton Keith Harrison, Scott Bukstein \\ DeVos Sport Business Management Program, College of Business \\ Administration, University of Central Florida, Orlando, USA \\ Email: scott.bukstein@ucf.edu, carlton.harrison@ucf.edu
}

Received July $7^{\text {th }}$, 2013; revised August $9^{\text {th }}$, 2013; accepted August 20 $0^{\text {th }}, 2013$

\begin{abstract}
Copyright (C) 2013 Carlton Keith Harrison, Scott Bukstein. This is an open access article distributed under the Creative Commons Attribution License, which permits unrestricted use, distribution, and reproduction in any medium, provided the original work is properly cited.
\end{abstract}

\begin{abstract}
This short report provides an overview and analysis of data on coaching mobility patterns in the National Football League (NFL). Previous studies in this area have generally focused on the effectiveness of the Rooney Rule (for example, analyzing the hiring process and proposing new strategies to increase the number of non-White head coaches) and comparing the win/loss records of White and non-White head coaches (for example, determining whether non-White coaches are provided with a meaningful opportunity to turn around a team with a losing record). This report focuses on whether Whites and non-Whites face systemic and socio-cultural access barriers after one or more stints as a head coach in the NFL. The findings of this study indicate that, historically, NFL teams have been reluctant to hire a non-White individual for a head coach, offensive coordinator or defensive coordinator position after a non-White individual has previously been fired or has resigned from a head coach position in the NFL.
\end{abstract}

Keywords: Mobility; Access Barriers; Occupational Mobility Patterns

\section{Introduction}

This short report provides a preliminary overview and analysis of data on coaching mobility patterns in the National Football League (NFL). Previous studies in this area have generally focused on the effectiveness of the Rooney Rule (for example, analyzing the hiring process and proposing new strategies to increase the number of non-White head coaches) and comparing the win/loss records of White and non-White head coaches (for example, determining whether non-White coaches are provided with a meaningful opportunity to turn around a team with a losing record). This report analyzes data provided by the NFL and focuses on whether Whites and non-Whites face systemic and socio-cultural access barriers after one or more stints as a head coach in the NFL. Stated differently, this report attempts to address whether Whites and/or non-Whites only have one opportunity to prove themselves, and therefore attention must focus on retention, career progression, continued access and "life after being a head coach" in addition to the Rooney's Rule noteworthy focus on initial entry/access for ethnic minorities. The authors of this short report hope that this report serves as a case study and platform for other scholars and practitioners to develop practical recommendations, policies, and processes to address the broader sociological issue relating to how intangible factors such as trust, implicit biases, informal networks, and perceived (in)competence impact occupational mobility.

\section{Concise Review of Literature on Occupational Mobility Patterns}

Social and behavioral scientists have developed various theories to explain status, power, and upward social mobility (see, e.g., Davis \& Moore, 1945; see also Zweigenhaft \& Domhoff, 2006). Previous research on occupational mobility patterns has generally focused on three approaches: the career or work history approach, the human capital approach (education and competencies), and the status attainment approach (social capital and mentors) (see Smith \& Abbott, 1983; see also Loy, 1969). Prior studies on occupational mobility of coaches has focused on geographical mobility patterns (see Sage \& Loy, 1978), structural barriers and management hierarchies (Braddock, Smith, \& Dawkins, 2012), and the importance of positioning individual coaching identities on specific hiring trees of influential employers and head coaches with icon status, access and opportunity (Brooks \& Althouse, 1993, 2000, 2007, 2013; Swaminathan, Wade, \& Schwabb, working paper). For example, results from quantitative analyses by Day \& McDonald (2010) demonstrated that social capital matters a great deal for promotions, but its impact is contingent on race; network connections to heterogeneous contacts (racially heterophilous ties, weak ties, and high status ties) appear to be more effective for African American coaches than for White coaches.

Other scholars have written about the social phenomena of racial stacking (determination of athlete playing position based on racial stereotypes) and centrality (relative distance to the center of the action on the playing field) to explain how race impacts the position an athlete plays (see, e.g., Phillips, 1983; Smith \& Harrison, 1996). Scholars have explained how athletes of color get "stacked" in "non-central" positions that require the smallest amounts of leadership qualities, interaction, and decision-making (see generally Yiannakis \& Melnick, 2001). These scholarly studies that focus on how stereotypes and implicit biases impact decisions with respect to athletes inform the cur- 
rent report's focus on occupational mobility of coaches. For instance, in a data-based study, Rosette, Leonardelli, \& Phillips (2008) found the following: 1) White business leaders are evaluated as more likely to succeed when such leaders are viewed as responsible for an organization's success; 2) White business leaders are considered more effective and typically experience better career advancement opportunities than racial minority leaders; 3) the lack of racial and ethnic minorities in top positions is due in part to leadership prototypes and leadership categorization theories; and 4) the unconscious and conscious label of "the White Standard" by evaluators means evaluators perceive successful leaders as White regardless of the evaluator's own race. Furthermore, Greene (2012) examined the "discourse of privilege" phenomenon that relies on rarely articulated subjective evaluation standards, which operates to exempt individuals who select candidates for head coach positions from contemporary norms of fairness and legitimacy.

\section{Methodology and Approach of Current Research Study}

This report investigated data regarding NFL head coach demographics, stint and mobility patterns from 1963-2012. This time period is used because 1963 serves as the first year that the NFL began to track relevant data on head coach mobility patterns. Based on the NFL database of human resources in terms of head coaches, these data were analyzed for mobility patterns. Report data is based on the start of the 2012 NFL season. Interim head coaches were not included in the data set with respect to determining the total number of people who have held head coaching positions in the NFL from 1963-2012. Attempts were made to verify the number of vacancies filled and individual separations, trajectories and occupational patterns of NFL head coaches based on the data provided by the NFL. If an individual was a head coach for multiple NFL teams, the report counts that coach one time in the data set because this report focuses on an analysis of access, opportunity and coaching mobility (i.e., the number of individuals who have held head coach positions) instead of the total number of head coach vacancies from 1963-2012.

\section{Findings and Results on NFL Coaching Mobility Patterns}

Based on data provided by the NFL, from 1963-2012 there have been 124 White head coaches in the NFL, 14 African American head coaches, and three Latino head coaches. Historically, the disparity and skewed representation between White head coaches in the NFL (87.9\%) and non-White head coaches (12.1\%) is indisputable over a fifty-year period (1963-2012). At the beginning of the 2012 NFL regular season, there were six non-White head coaches (18.8\% of head coaches), as compared with 26 White head coaches (81.2\% of head coaches). At the time of publication of this report, there were only four nonWhite head coaches (12.5\% of head coaches in the NFL).

The findings of this study indicate that, historically, NFL teams have been reluctant to hire a non-White individual for a head coach, offensive coordinator or defensive coordinator position after a non-White individual has previously been fired or has resigned from a head coach position in the NFL. For example, after separating (i.e., being fired or resigning) from a first head coach position, seven non-White individuals (41.2\% of the
17 total non-White head coaches from 1963-2012) have received (and accepted) a second head coach opportunity in the NFL. However, since 2007 only one non-White individual, Romeo Crennel, has received (and accepted) a second head coach opportunity. After separating from a second head coach position, only one non-White coach, Tom Flores, has received (and accepted) a third opportunity to be the head coach of an NFL team. Not a single non-White coach has had a fourth opportunity to be the head coach of an NFL team.

After separating from a first head coach position, 46 White individuals (37.1\% of the 124 total White head coaches from 1963-2012) have received (and accepted) a second head coach opportunity, as compared with seven non-White individuals. Twelve White coaches have received (and accepted) a third opportunity to be the head coach of an NFL team, as compared with only one non-White coach, Tom Flores. Three White coaches (Bill Parcells, Wade Phillips, and Marty Schottenheimer) have had a fourth opportunity to be the head coach of an NFL team, as compared with zero non-White individuals.

After separating from a first head coach position, 21 White individuals have held defensive coordinator positions and 19 White individuals have held offensive coordinator positions. After separating from a first head coach position, one nonWhite individual (Romeo Crennel) has held the defensive coordinator position and one non-White individual (Tom Fears) has been an offensive coordinator. It is important to note that only two non-White individuals (Romeo Crennel and Tom Fears) have accepted an offensive coordinator or defensive coordinator position after one stint as a head coach in the NFL, and no non-White individual has held an offensive coordinator position after one stint as an NFL head coach since Tom Fears made that transition in the early $1970 \mathrm{~s}^{1}$.

Three White individuals have held defensive coordinator positions and three White individuals have held offensive coordinator positions after separating from a second head coach position. Only one non-White individual (Ray Rhodes) has held a defensive coordinator position and zero non-White coaches have held an offensive coordinator position after separating from a second stint as a head coach in the NFL.

Since 1980, approximately 30 individuals who have served as head coaches in the NFL have subsequently accepted a head coach position with a college football team in the Football Bowl Subdivision (FBS). All of these individuals have been White coaches. Stated differently, zero non-White individuals have successfully transitioned from a former NFL head coach to a college football head coach since 1980 . It is important to note that there is no reliable data with respect to how many non-White individuals have pursued (but were not offered and/ or did not accept) these college head coach positions after at least one stint as a head coach in the NFL.

Fourteen African American individuals have been head coaches in the NFL since 1963. Six additional African American individuals have held interim head coach positions (i.e., these individuals were head coaches for a part of an NFL season) but were not offered the head coach position for the following full NFL season. Only five NFL teams have hired two African American head coaches from 1963-2012. No NFL team has hired three African American head coaches. Also, the Indian-

${ }^{1}$ Report data is based on the beginning of the 2012 NFL regular season. Jim Caldwell, an African American who was previously the head coach of the Indianapolis Colts, was named Offensive Coordinator of the Baltimore Ravens during the 2012 NFL season. 
apolis Colts became the first (and only) NFL team to hire African American head coaches back-to-back when the Colts hired Jim Caldwell to succeed Tony Dungy in 2009.

Eight NFL head coaches were fired shortly after the end of the 2012 NFL regular season. Six of these head coaches were White individuals, and two were non-White coaches. As of the time of publication of this report, four of the six White individuals had already accepted another NFL coaching-related position (one as a head coach and three as offensive coordinators), but neither of the two non-White individuals had been named to a head coach or coordinator position.

Please refer to Tables 1-3 below for an overview of primary data findings.

\section{Discussion and Conclusion}

A 2002 study commissioned by the late Johnnie Cochran, Jr. and prominent employment discrimination lawyer Cyrus Mehri, conducted by Dr. Janice Madden, looked directly at the performance statistics of African American head coaches in the NFL compared to those of White coaches; the study found that African American head coaches averaged 1.1 more wins per season, led their teams to the playoffs $67 \%$ of the time compared to $39 \%$ of the time for White coaches, averaged 2.7 more wins in their first season and, in their final seasons, terminated African American coaches averaged 1.3 more wins than White coaches who were also terminated (see Greene, 2012). Notwithstanding this historical data on winning percentages based on the race of a head coach, the current study found that, in terms of occupational mobility, the second and third chances for non-Whites to continue coaching at the positions of head coach, offensive coordinator and defensive coordinator have

Table 1.

Race of NFL head coaches (1963-2012).

\begin{tabular}{ccc}
\hline & Number of Coaches & Percent \\
\hline Non-White & 17 & $12.1 \%$ \\
White & 124 & $87.9 \%$ \\
Total & 141 & $100.0 \%$ \\
\hline
\end{tabular}

Table 2.

NFL opportunity after first head coach position.

\begin{tabular}{ccc}
\hline & \multicolumn{2}{c}{ Race } \\
\cline { 2 - 3 } & Non-White & White \\
\hline NFL Head Coach & 7 & 46 \\
NFL Offensive Coordinator & 1 & 19 \\
NFL Defensive Coordinator & 1 & 21 \\
Totals & $\mathbf{9}$ & $\mathbf{8 6}$ \\
\hline
\end{tabular}

Table 3.

NFL opportunity after second head coach position.

\begin{tabular}{ccc}
\hline & \multicolumn{2}{c}{ Race } \\
\cline { 2 - 3 } & Non-White & White \\
\hline NFL Head Coach & 1 & 12 \\
NFL Offensive Coordinator & 0 & 3 \\
NFL Defensive Coordinator & 1 & 3 \\
Totals & $\mathbf{2}$ & $\mathbf{1 8}$ \\
\hline
\end{tabular}

been inequitable in comparison to Whites in the NFL from 1963-2012.

The findings of the study in this report underscore and uncover the complexity of organizational nuances that may influence the final hiring decisions at the professional and collegiate levels and determine the fate of non-White coaches to maneuver the hierarchies of leadership positions. Previous analysis on this concept of fewer career opportunities has focused on African American quarterbacks and the phenomenon of racial stacking (Edwards, 1973; Lapchick, 1991; Smith, 2007), as well as the lack of ethnic minorities in other major professional sport leagues (e.g., Major League Baseball). In addition, researchers such as Professor Katherine Phillips and colleagues challenge scholars and practitioners to examine this issue on a deeper level beyond perceptions, policy and awareness. Phillips et al. have developed a theory-based argument that supports the current report's research findings with respect to whether variables such as "institutional inequality" (Davis \& Moore, 1945: p. 243; see also Acker, 2006) and "membership in powerful coaching families” (Greene, 2012: p. 131) have more of an impact in the hiring and evaluation processes than do the substantive skill sets of individuals.

Phillips and her colleagues were inspired to research this topic after the fate of college head football coaches like Ty Willingham at Notre Dame, who compiled a 21-15 record but was fired after three years on a five-year contract, thereby becoming the first coach in the university's history at the time to have his contract terminated in the middle of his tenure. Phillips explained, "We had a few ideas before the project, but the project started shaping itself. We started thinking that African Americans are not getting the credit they deserve; they do not always have the doors opened; and when they get there they are evaluated differently” (Kellogg Insight, 2008: p. 1). Similar in part to this report's focus, these researchers asked the question does the phenomenon of discrimination differences and different evaluative criteria with respect to job performance happen in business and whether one "can one show that these differences exist and have an impact on people's ability to ascend to leadership positions and stay there?” (Kellogg Insight, 2008: p. 1).

Whites and non-Whites experience different mobility patterns of success and failure as they move from organization to organization in the NFL (see Rosette et al., 2008). While the Rooney Rule has been effective in allowing ethnic minority candidates more initial access than was previously realized at the time-the culture of NFL male networks, cryonism (i.e., showing favoritism to friends and colleagues without regard to actual competencies and qualifications), and the "who knows you" culture requires that a serious analysis of the situation continues to occur. For now, the data in the current report affirm that some standard is in place for certain coaches to jump from team to team in head coaching or coordinator roles. Is that standard racist? That should not be the focus-the focus should be on changing the culture and figuring out why the same attributes that normally dictate "the reshuffling effect" for White coaches does not transfer over for non-White coaches with the same pedigree (or even better pedigree) after their first or second separation from a head coaching position in the NFL. The data in the current report support the research by several colleagues mentioned earlier in this report that non-White coaches face a different reality in terms of "playing the game" and "staying in the game." 
The public discourse in terms of fans and outside observers of the NFL's diversity and inclusion issues and policies such as the Rooney Rule remains limited, ill-informed and in need of some factual information on how diversity is defined, categorized and analyzed. As is often the case, numerous fans perceive that contemporary society is "post-racial" and that there is no need to address racial, gender and other social issues that impact sport in society. The high representation of non-White football players in particular distorts the public perception that equality has been realized. Access to leadership and top management roles in professional and college sport is not the same as access to physical participation at the competitive levels on the field of play. In the future, a strong push to strategically and systematically educate fans of the NFL could help contribute to a national and global culture that understands the true evidence relating to equality and the true meaning of equality.

The Rooney Rule has unquestionably helped to shape a culture of opportunity in terms of those individuals that make it to the final interview process from a wider candidate pool. However, while the Rooney Rule "combats unconscious bias and increases the chances of selecting the best person for the job" (Proxmire, 2008: p. 9), there remains a need to improve both the policy and the process. The Rooney Rule has provided many non-White head coach candidates with access to a meaningful interview. The next step is to provide non-Whites with access to information about the culture of the NFL and with access to the powerful formal and informal networks (that is, social capital) that impact whether an individual might have a second or third opportunity in the NFL. The Rooney Rule may enable a non-White individual to have an opportunity to secure that initial head coach position, but intangible factors such as trust and perceived competence may have even more of an impact on future occupational mobility (second and third coaching opportunities). Therefore, in addition to working to increase the number of non-Whites who make hiring decisions (team owners and general managers), it is imperative to work on improving "the perception of competence" of non-White sport business professionals by both Whites and non-Whites (Shropshire, 1996: pp. 129-30). Stated differently, even if there is an increase in non-White general managers and team owners, negative race consciousness associated with the coaching capabilities of non-Whites may still exist and persist (see Shropshire, 1996). Strategic diversity management is a business imperative (see Thomas, 2010), as a more diverse and inclusive (and informed) workforce will make the NFL an even stronger organization and brand.

\section{REFERENCES}

Acker, J. (2006). Class questions, feminist answers. New York: Routledge.
Braddock, J. H., Smith, E., \& Dawkins, M. (2012). Race and pathways to power in the National Football League. American Behavioral Scientist, 56, 711-727. http://dx.doi.org/10.1177/0002764211433802

Brooks, D., \& Althouse, R. (1993, 2000, 2007, 2013). Racism in college athletics: The African-American athlete's experience. Morgantown, West Virginia: Fitness Information Technology.

Cochran Jr., J., \& Mehri, C. (2002). Black coaches in the NFL: Superior performance, inferior opportunities. Washington DC: Mehri \& Skalet, PLLC.

Davis, K., \& Moore, W. E. (1945). Some principles of stratification. American Sociological Review, 10, 242-249. http://dx.doi.org/10.2307/2085643

Day, J. C., \& McDonald, S. (2010). Not so fast my friend: Social capital and the race disparity in promotions among college football coaches. Sociological Spectrum: Mid-South Sociological Association, 30, 138-158. http://dx.doi.org/10.1080/02732170903495937

Edwards, H. (1973). Sociology of sport. Homewood, IL: Dorsey Press.

Greene, L. (2012). Head football coaches: Ending the discourse of privilege. Wake Forest Journal of Law \& Policy, 2, 115-142.

Kellogg Insight (2008). Transparent barriers. Evanston: Kellogg School of Management at Northwestern University.

Lapchick, R. E. (1991). Five minutes to midnight: Race and sport in the 1990s. Lanham, Maryland: Rowman \& Littlefield.

Loy Jr., J. W. (1969). The study of sport and social mobility. In G. S. Kenyon (Ed.), Aspects of contemporary sport sociology (pp. 101119). Madison, Wisconsin: Athletic Institute.

Phillips, J. C. (1983). Race and career opportunities in Major League Baseball: 1960-1980. Journal of Sport and Social Issues, 7, 1-17. http://dx.doi.org/10.1177/019372358300700201

Proxmire, D. (2008). Coaching diversity: The rooney rule, its application and ideas for expansion. American Constitution Society for Law and Policy, 1-9.

Rosette, A. S., Leonardelli, G. J., \& Phillips, K. W. (2008). The white standard: Racial bias in leader categorization. Journal of Applied Psychology, 93, 758-777. http://dx.doi.org/10.1037/0021-9010.93.4.758

Sage, G., \& Loy, J. (1978). Geographical mobility patterns of college coaches. Journal of Contemporary Ethnography, 7, 253-280. http://dx.doi.org/10.1177/089124167800700208

Shropshire, K. (1996). In black and white: Race and sports in America. New York: New York University Press.

Smith, D. R., \& Abbott, A. (1983). A labor market perspective on the mobility of college football coaches. Social Forces, 61, 1147-1167. http://dx.doi.org/10.2307/2578284

Smith, E. (2007). Race, sport and the American dream. Durham, North Carolina: Carolina Academic Press.

Smith, E., \& Harrison, C. K. (1996). Stacking in major league baseball. Journal of African American Men, 2, 113-129. http://dx.doi.org/10.1007/s12111-996-1006-3

Swaminathan, A., Wade, J., \& Schwabb, A. (2013) Employment affiliation networks and career mobility among NFL coaching staff, 19852008. Working Paper. Atlanta: Emory University.

Thomas, R. (2010). World class diversity management: A strategic approach. San Francisco: Berrett-Koehler Publishers.

Yiannakis, A., \& Melnick, M. (2001). Contemporary issues in socialogy of sport (5th ed.). Champaign, IL: Human Kinetics Publishers.

Zweigenhaft, R., \& Domhoff, W. (2006). Diversity in the power elite. New York: Rowman \& Littlefield. 